\title{
An Improved Magnetic Field Simulator - MAGFLD
}

\author{
TK Ghosh, RG Carter*, Vinay Yadav, Yukta Joshi, Rishma Gupta, \\ Aakanksha Srivastava, R Vishwanath and SN Joshi
}

Central Electronics Engineering Research Institute (CEERI), Pilani (Raj) - 333 031, India, Email: tkghosh@,ceeri.ernet.in, Phone: +91 1596 252358, Fax: +91 1596242294

*Engineering Department, Lancaster University, LA1 4YR, UK, r.carter@lancaster.ac.uk

An improved two-dimensional simulator MAGFLD has been developed at CEERI, which is useful for the design and simulation of periodic permanent magnet (PPM) focusing system for linear beam tubes. At present, input is possible only through the input file, which is very simple and user friendly. A complete PPM circuit is generated using the coordinates of first pole piece, first magnet, gun adapter (if the structure is a-periodic) and the region of computation. Small mesh units of either square or rectangular shapes can be used with mesh refinement capability in one or more regions in any or both directions for better accuracy of the solution. Materials with different magnetic permeability can be modeled by defining a characteristic value for each mesh point of the geometry. The effective potential value at each point in the region of interest is calculated based on the vector potential model [1] by using the 5-point finite difference method (FDM) and the solution is achieved by over relaxation technique for faster convergence. This package has an interface with EGUN [2] to model the electron gun and collector under the influence of magnetic field. Versatile color graphics are capable of plotting both axial magnetic field and flux lines along with the magnetic circuit. The package is written in ' $\mathrm{C}$ ' language and it is capable of running in different operating systems like DOS, Linux, DEC-OSF and DEC-VMS.

MAGFLD has been validated against some published data and experimental results. A comparison between the theoretical and experimental results is shown in the TABLE I. Here, the peak axial magnetic field has been simulated for a stack of one period length (assumed infinitely long periodic structure). The experimental value of the axial magnetic field has been obtained for a PPM stack of five-period length of three different TWTs developed at CEERI. It is observed from the table that the theoretical results are very close to the experimental values. The fringing field has also been measured and compared with the simulated results in TABLE II, which are in good agreement. In Fig. 1 the simulated magnetic field has been compared with the measured values in the electron gun region of a $6-18 \mathrm{GHz}$ gain and phase matched $40 \mathrm{~W}$ mini-TWT. This shows a very good agreement between the theoretical and the practical values. Finally this package has been used for profiling the magnetic field in the gun region of a Ku-band space TWT [3]. The electron beam has been traced from the electron gun to the collector under dc conditions using a gun collector module [4], which is shown in Fig. 2. The modules of the package and its capabilities will be presented in detail during the conference.

\section{REFERENCES}

[1] Y Morizumi, "Computer-Aided Design of an Axially Symmetrical Magnetic Circuit and its Application to Electron-Beam Focussing Devices", IEEE Trans on Electron Devices, vol. ED-19, No. 6, 783 (1972).

[2] W B Herrmannsfeldt, "EGUN-an Electron Optics and Gun Design Program," SLAC, 50 (1998)

[3] V Srivastava, T K Ghosh, R K Sharma, A K Sinha, V V P Singh, "Preliminary Design Report of Ku-band $140 \mathrm{~W}$ Space TWT", CEERI, 70 (2003)

[4] TK Ghosh, Rishma Gupta, and V Srivastava, "Development of a Gun-Collector Module for a Space TWT", CODEC-04, (2004) to be presented. 
TABLE I: Comparison of theoretical and practical results of peak magnetic field

\begin{tabular}{|l|c|c|c|c|}
\hline \multirow{2}{*}{ Type of TWT } & \multicolumn{2}{|c|}{$\begin{array}{c}\text { Peak Mag. Field in Circuit } \\
\text { (Gauss) }\end{array}$} & \multicolumn{2}{c|}{$\begin{array}{c}\text { Peak Mag. Field for Free Magnet } \\
\text { (Gauss) }\end{array}$} \\
\cline { 2 - 5 } & Simulated & Measured & Simulated & Measured \\
\hline CC-TWT & 2475 & 2400 & 780 & 760 \\
\hline Space TWT & 1125 & 1100 & 740 & 710 \\
\hline Mini-TWT & 2100 & 2000 & 1030 & 1000 \\
\hline
\end{tabular}

TABLE II: Comparison of theoretical and practical results of free magnets

\begin{tabular}{|l|c|c|c|c|}
\hline \multirow{3}{*}{ Type of TWT } & \multicolumn{2}{|c|}{$\begin{array}{c}\text { Axial Peak Mag. Field } \\
\text { (Gauss) }\end{array}$} & $\begin{array}{c}\text { Axial Peak Mag. Field after Zero Crossing } \\
\text { (Gauss) }\end{array}$ \\
\cline { 2 - 5 } & Simulated & Measured & Simulated & Measured \\
\hline Morizumi [1] & 600 & 630 & 121 & 120 \\
\hline Mini & 1030 & $975 \pm 50$ & 206 & 192 \\
\hline
\end{tabular}

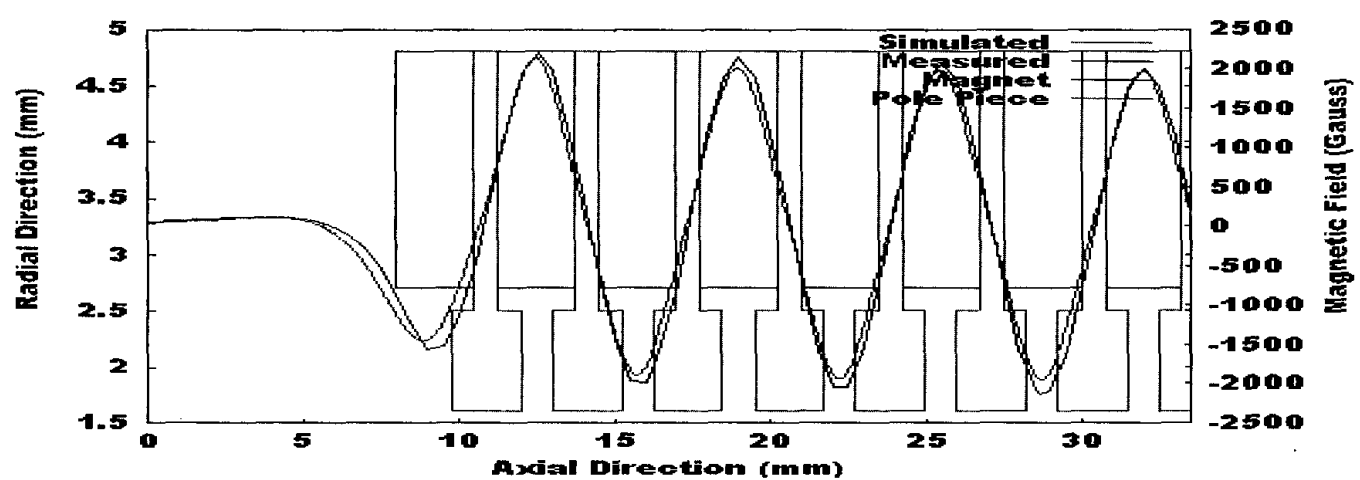

Fig. 1: Comparison between the simulated and the measured magnetic field in the gun region of mini-TWT

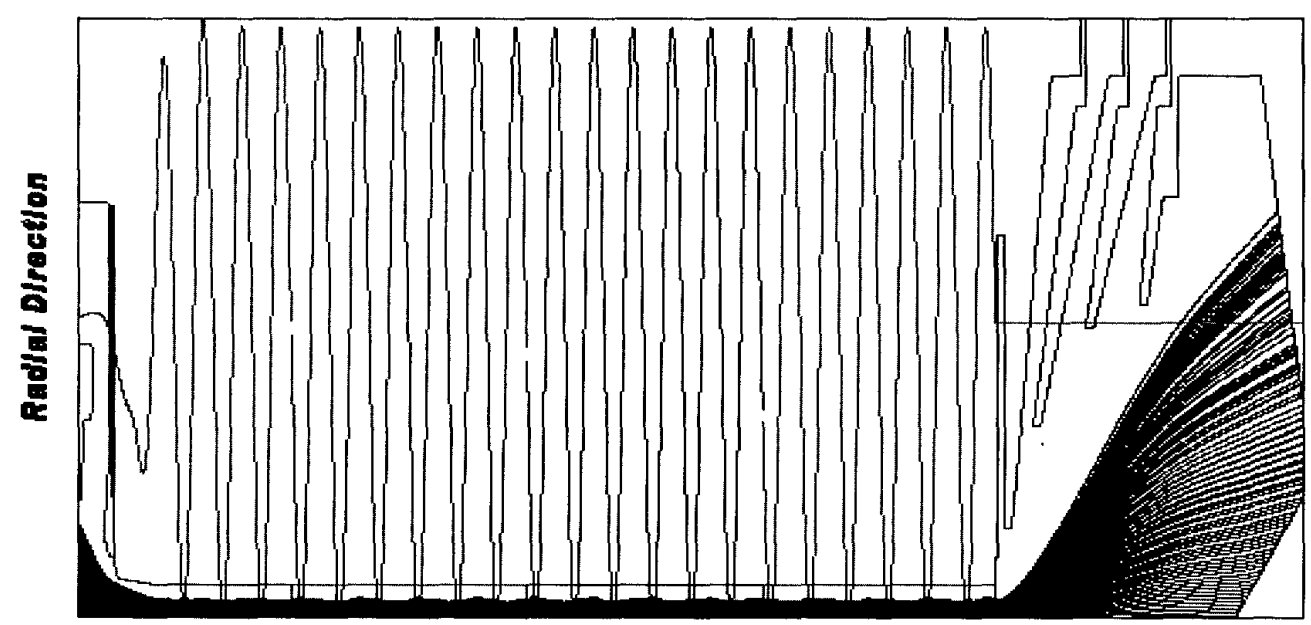

Axiar Ditrectrom

Fig. 2: Gun collector module of a Ku-band $140 \mathrm{~W}$ space TWT showing the PPM focused dc electron beam 